\title{
Super-Resolution with Multiselective Contourlets
}

\author{
Mohamed El Aallaoui ${ }^{1}$, Abdelwahad Gourch ${ }^{2}$ \\ ${ }^{1}$ Laboratory of Mathematical Engineering (LINMA), Department of Mathematics and Computer Science, \\ Faculty of Sciences, Eljadida, Morocco \\ ${ }^{2}$ Faculté des Sciences Juridiques, Économiques et Sociales de Ain Sebaâ, Casablanca, Morocco \\ Email: m_elaallaoui@yahoo.fr, agourch2002@yahoo.fr
}

Received July 18, 2012; revised September 19, 2012; accepted October 10, 2012

\begin{abstract}
We introduce a new approach to image super-resolution. The idea is to use a simple wavelet-based linear interpolation scheme as our initial estimate of high-resolution image; and to intensify geometric structure in initial estimation with an iterative projection process based on hard-thresholding scheme in a new angular multiselectivity domain. This new domain is defined by combining of laplacian pyramid and angular multiselectivity decomposition, the result is multiselective contourlets which can capture and restore adaptively and slightly better geometric structure of image. The experimental results demonstrate the effectiveness of the proposed approach.
\end{abstract}

Keywords: Super-Resolution; Laplacian Pyramid; Angular Multiselectivity; Multiselective Contourlets; Anti-Aliasing Filer; Sparsity Constraint; Iterative Projection

\section{Introduction}

In most digital imaging applications, high-resolution images or videos are usually desired for later image processing and analysis. The desire for high resolution stems from two principal application areas: improvement of pictorial information for human interpretation; and helping representation for automatic machine perception $[1,2]$. Image resolution describes the details contained in an image, the higher the resolution, the more image details $[1,3]$. Super-resolution is techniques that construct highresolution images from several observed low-resolution images, thereby increasing the high-frequency components and removing the degradations caused by the imaging process of the low-resolution camera. The basic idea behind super-resolution is to combine the non-redundant information contained in multiple low-resolution frames to generate a high-resolution image. The superresolution (SR) reconstruction of a digital image can be classified in many different ways: SR in spatial domain [4,5], SR in the Frequency Domain [6,7], Statistical Approaches [8,9], and Interpolation-Restoration $[1,10]$. In this last context, can be distinguished two categories, linear and nonlinear interpolation methods.

Linear interpolation methods, such as bilinear, bicubic and cubic spline [11,12], edge-sensitive filter [13], blurring and ringing effects because they do not utilize any information relevant to geometric structure of image $[14,15]$. Nonlinear interpolation methods incorporate more adaptive image models and priori knowledge which often improve linear interpolators. Many approaches have been designed for addressing this task in recent years. We may cite for instance, Soft-decision Adaptive Interpolation (SAI) [16], Sparse Mixing Estimators (SME) [17], Iterative Projection [18], $\cdots$

The SAI approach has been improved by Zhang and $\mathrm{Wu}$, by using an interpolator adapted to local covariance image based on autoregressive image models optimized over image blocks. This approach can be more accurate, it is much more demanding in computation and memory resources. The SME approach proposed by Mallat and $\mathrm{Yu}$, computes a high-resolution estimator by mixing adaptively a family of linear estimators corresponding to different priors. Sparse mixing weights are calculated over blocks of coefficients in a frame providing a sparse signal representation. Mueller and $\mathrm{Lu}$ have proposed an iterative interpolation method based on the wavelet and contourlet transforms $[19,20]$. In this approach, the contourlet transform improves the visual quality of resulting images, by intensification of the geometric structure on the wavelet linear interpolation. This geometric structure is well represented by contourlets with variable angular selectivity [21]. However, the contoulets represent the image geometry with the same angular selectivity $[19,20]$. In order to overcome this limitation of representation of geometric structure in this iterative approach, we have increased the sensitivity of angular selectivity of contourlets. Our idea is based on a simple wavelet-based linear interpolation scheme as our initial estimate; and an iterative projection process based on hard-thresholding 
scheme in a new angular multiselectivity domain. This new domain is defined by combining of laplacian pyramid and an angular multiselectivity decomposition. The result is new multiselective contourlets, which can represent the different structures of the image geometry.

The paper is organized as follows. In Sections 2 and 3, we discuss the new multiselective contourlets, and we will show how these multiselective contourlets can provide a new degree of freedom to describe adaptively the different structures of the image geometry. Our multiselective contourlets algorithm for image super-resolution is described in the Section 4. We report the results of our experiments in Section 5 and conclude the paper in Section 6.

\section{Laplacian Pyramid}

The Laplacian Pyramid ( $L P$ ) was first proposed in [22] as a new technique for compression image. To achieve high compression, it removes image correlation by combining predictive and transform coding techniques.

In the Laplacian Pyramid decomposition at each level the original image happens in a high-pass and a low-pass filters, the resulting is a downsampled low-pass version of the original image, and of difference between the original image and the prediction.

Under certain regularity conditions, the low-pass filter $g$ in the iterated $L P$ uniquely defines a unique scaling function $\phi(t) \in L^{2}\left(\mathbb{R}^{2}\right)$ that satisfies the following twoscale equation $[23,24]$

$$
\phi(t)=2 \sum_{n \in \square} g[2] \phi(2 t-n) .
$$

Let

$$
\phi_{j, n}(t)=2^{-j} \phi\left(\frac{t-2^{j} n}{2^{j}}\right), j \in \square, n \in \square^{2} .
$$

Then the family $\left\{\phi_{j, n}\right\}_{n \in \square^{2}}$ is an orthonormal basis for an approximation subspace $V_{j}$ at the scale $2^{j}$. Furthermore, $\left\{V_{j}\right\}_{j \in \square}$ provides a sequence of multiresolution nested subspaces $\cdots V_{2} \in V_{1} \in V_{0} \in V_{-1} \in V_{-2} \cdots$, where $V_{j}$ is associated with a uniform grid of intervals $2^{j} \times 2^{j}$ that characterizes image approximation at scale $2^{j}$. The difference images in the $L P$ contain the details necessary to increase the resolution between two consecutive approximation subspaces. Therefore, the difference images live in a subspace $W_{j}$ that is the orthogonal complement of $V_{j}$ in $V_{j-1}$, or

$$
V_{j-1}=V_{j} \oplus W_{j} \text {. }
$$

The $L P$ can be considered as an oversampled filter bank where each polyphase component of the difference signal comes from a separate filter bank channel like the coarse signal [25]. Let $F_{i}(z), 0 \leq i \leq 3$ be the synthesis filters for these polyphase components. Note that these synthesis filters are high-pass filters. As for wavelets, we associate with each of these filters a continuous function $\varphi^{i}(t)$ where

$$
\varphi^{i}(t)=2 \sum_{n \in \square^{2}} f_{i} \phi(2 t-n) .
$$

Proposition 2.1 ([25]): let

$$
\varphi_{j, n}^{i}(t)=2^{-j} \varphi^{i}\left(\frac{t-2^{j} n}{2^{j}}\right), j \in \square, n \in \square^{2} .
$$

Then, for scale $2^{j},\left\{\varphi_{j, n}^{i}\right\}_{0 \leq i \leq 3, n \in \square^{2}}$ is a tight frame for $W_{j}$.

Since $W_{j}$ is generated by four kernel functions (similar to multi-wavelets), in general it is not a shiftinvariant subspace. Nevertheless, we can simulate a shiftinvariant subspace by denoting

$$
\psi_{j, 2 n+k_{i}}(t)=\varphi_{j, n}^{i}(t), 0 \leq i \leq 3 .
$$

where $k_{i}$ are the coset representatives for downsampling by 2 in each dimension

$$
\begin{aligned}
& k_{0}=(0,0)^{\mathrm{T}}, k_{1}=(1,0)^{\mathrm{T}}, \\
& k_{2}=(0,1)^{\mathrm{T}}, k_{3}=(1,1)^{\mathrm{T}} .
\end{aligned}
$$

With this notation, the family $\left\{\psi_{j, n}\right\}_{n \in \square^{2}}$ associated to an uniform grid of intervals $2^{j-1} \times 2^{j-1}$ on $\mathbb{R}^{2}$ provides a tight frame for $W_{j}$ [25]. Then the family $\left\{\psi_{j, n}\right\}_{n \in \square^{2}}$ suffices the following equality:

$$
\sum_{n \in \square^{2}}\left|\left\langle\psi_{n, j} \mid f\right\rangle\right|^{2}=\|f\|^{2}, \quad \forall f \in W_{j} .
$$

\section{Multiselective Contourlets}

In this section we propose the multiselective contourlets defined by combining of laplacian pyramid and an angular multiselectivity decomposition, and we will show how these new contourlets can provide a new degree of freedom to describe adaptively the different structures of the image geometry.

We consider $2 \pi$-periodic function $\tau_{\alpha}$ defined by

$$
\tau_{\alpha}(\theta)=\left\{\begin{array}{cl}
\gamma\left(\frac{\theta-\alpha}{\alpha}\right), & \theta \in[0,2 \alpha] ; \\
1, & \theta \in[2 \alpha, \pi] ; \\
\gamma\left(\frac{\pi+\alpha-\theta}{\alpha}\right), & \theta \in[\pi, \pi+2 \alpha] ; \\
0, & \theta \in[\pi+2 \alpha, 2 \pi] .
\end{array}\right.
$$


where $\alpha \in[0, \pi]$ and the function $\gamma$ is defined in $[-1,1]$ and satisfies the following property:

$$
\gamma^{2}(t)+\gamma^{2}(-t)=1 \text {. }
$$

For $L \in \mathbb{N}^{*}$ and $\alpha=\frac{\pi}{2^{L}}$, we create $2^{l}$ different $2 \pi$ periodic functions $\eta_{l, m}$ indexed by $0 \leq m<2^{l}$ for any $l \in\{0, \cdots, L\}$ defined by:

$$
\begin{aligned}
\eta_{0,0}(\theta)= & 1, \\
\eta_{l+1,2 m}(\theta)= & \eta_{l, m}(\theta) \tau_{\alpha} \\
& \cdot\left(\theta-\frac{(2 m+1) \pi}{2^{l}}\right), \\
\eta_{l+1,2 m+1}(\theta)= & \eta_{l, m}(\theta) \tau_{\alpha} \\
& \cdot\left(\theta-\frac{(2 m+1) \pi}{2^{l}}-\pi\right) .
\end{aligned}
$$

By the laplacian pyramidc wavelets $\psi_{j, n}$ defined in the previous section and for each subspace $W_{j}$, we construct a new contourlets whose Fourier transforms are:

$$
\hat{\psi}_{j, n, l, m}(\boldsymbol{k})=\hat{\psi}_{j, n}(\boldsymbol{k}) \eta_{l, m}(\theta),
$$

where $\theta=\arg \boldsymbol{k}$.

Proposition 3.1 for any $l \in\{1, \cdots, L\}$

$$
\begin{aligned}
& \eta_{l, 0}(\theta) \\
& =\left\{\begin{array}{cl}
\gamma\left(\frac{\theta-\alpha}{\alpha}\right), & \theta \in[0,2 \alpha] ; \\
1, & \theta \in\left[2 \alpha, \frac{\pi}{2^{l-1}}\right] ; \\
\gamma\left(\frac{\frac{\pi}{2^{l-1}}+\alpha-\theta}{\alpha}\right), & \theta \in\left[\frac{\pi}{2^{l-1}}, \frac{\pi}{2^{l-1}}+2 \alpha\right] ; \\
0, & \theta \in\left[\frac{\pi}{2^{l-1}}+2 \alpha, 2 \pi\right] .
\end{array}\right.
\end{aligned}
$$

and $\forall m \in\left\{0, \cdots, 2^{l}-1\right\}$

$$
\begin{aligned}
\hat{\psi}_{j, n, l, m}(\boldsymbol{k}) & =\hat{\psi}_{j, n}(\boldsymbol{k}) \eta_{l, m}(\theta) \\
& =\hat{\psi}_{j, n}(\boldsymbol{k}) \eta_{l, 0}\left(\theta-m \frac{2 \pi}{2^{l}}\right) .
\end{aligned}
$$

\section{Proof}

According to the expression (9) of the function $\tau_{\alpha}$, one have for any $l \in\{1, \cdots, L\}$ :

$$
\begin{aligned}
& \tau_{\alpha}\left(\theta-\frac{\pi}{2^{l}}\right) \\
& 0, \quad \theta \in\left[0, \frac{\pi}{2^{l}}\right] \text {; } \\
& \gamma\left(\frac{\theta-\frac{\pi}{2^{l}}-\alpha}{\alpha}\right), \quad \theta \in\left[\frac{\pi}{2^{l}}, \frac{\pi}{2^{l}}+2 \alpha\right] \\
& =\left\{1, \quad \theta \in\left[\frac{\pi}{2^{l}}+2 \alpha, \frac{\pi}{2^{l}}+\pi\right]\right. \text {; } \\
& \gamma\left(\frac{\pi+\frac{\pi}{2^{l}}+\alpha-\theta}{\alpha}\right), \theta \in\left[\frac{\pi}{2^{l}}+\pi, \frac{\pi}{2^{l}}+\pi+2 \alpha\right] \\
& 0, \quad \theta \in\left[\frac{\pi}{2^{l}}+\pi+2 \alpha, 2 \pi\right] . \\
& \tau_{\alpha}\left(\theta-\pi-\frac{\pi}{2^{l}}\right) \\
& \left\{\begin{array}{cl}
1, & \theta \in\left[0, \frac{\pi}{2^{l}}\right] ; \\
\gamma\left(\frac{\frac{\pi}{2^{l}}+\alpha-\theta}{\alpha}\right), & \theta \in\left[\frac{\pi}{2^{l}}, \frac{\pi}{2^{l}}+2 \alpha\right] ; \\
0, & \theta \in\left[\frac{\pi}{2^{l}}+2 \alpha, \frac{\pi}{2^{l}}+\pi\right] ; \quad \tau \\
\gamma\left(\frac{\theta-\pi-\frac{\pi}{2^{l}}-\alpha}{\alpha}\right), & \theta \in\left[\frac{\pi}{2^{l}}+\pi, \frac{\pi}{2^{l}}+\pi+2 \alpha\right] ; \\
1, & \theta \in\left[\frac{\pi}{2^{l}}+\pi+2 \alpha, 2 \pi\right] .
\end{array}\right.
\end{aligned}
$$

We shall now prove that for any $l \in\{1, \cdots, L\}$

$$
\eta_{l, 0}(\theta)=\left\{\begin{array}{cc}
\gamma\left(\frac{\theta-\alpha}{\alpha}\right), & \theta \in[0,2 \alpha] ; \\
1, & \theta \in\left[2 \alpha, \frac{\pi}{2^{l-1}}\right] ; \\
\gamma\left(\frac{\frac{\pi}{2^{l-1}}+\alpha-\theta}{\alpha}\right), & \theta \in\left[\frac{\pi}{2^{l-1}}, \frac{\pi}{2^{l-1}}+2 \alpha\right] ; \\
0, & \theta \in\left[\frac{\pi}{2^{l-1}}+2 \alpha, 2 \pi\right] .
\end{array}\right.
$$

Let's prove this by induction: Since $\eta_{1,0}(\theta)=\eta_{0,0}(\theta) \tau_{\alpha}(\theta-\pi-\pi)=\tau_{\alpha}(\theta)$, the function $\eta_{1,0}$ 
expressed as (19). Now assume that for a fixed $l$, the function $\eta_{l, 0}$ expressed as (19). The inclusion of this induction hypothesis and Equation (18) in the expression (12) gives:

$$
\eta_{l+1,0}(\theta)=\left\{\begin{array}{cc}
\gamma\left(\frac{\theta-\alpha}{\alpha}\right), & \theta \in[0,2 \alpha] ; \\
1, & \theta \in\left[2 \alpha, \frac{\pi}{2^{l}}\right] ; \\
\gamma\left(\frac{\frac{\pi}{2^{l}}+\alpha-\theta}{\alpha}\right), & \theta \in\left[\frac{\pi}{2^{l}}, \frac{\pi}{2^{l}}+2 \alpha\right] ; \\
0, & \theta \in\left[\frac{\pi}{2^{l}}+2 \alpha, 2 \pi\right] .
\end{array}\right.
$$

This last result completes the proof of the induction.

The insertion of the expressions (17) and (19) in Equation (13) shows that: for any $l \in\{1, \cdots, L\}$

$$
\eta_{l, 1}(\theta)=\left\{\begin{array}{cc}
0, & \theta \in\left[0, \frac{\pi}{2^{l-1}}\right] ; \\
\gamma\left(\frac{\theta-\frac{\pi}{2^{l-1}}-\alpha}{\alpha}\right), & \theta \in\left[\frac{\pi}{2^{l-1}}, \frac{\pi}{2^{l-1}}+2 \alpha\right] ; \\
1, & \theta \in\left[\frac{\pi}{2^{l-1}}+2 \alpha, \frac{\pi}{2^{l-2}}\right] ; \\
\gamma\left(\frac{\frac{\pi}{2^{l-2}}+\alpha-\theta}{\alpha}\right), & \theta \in\left[\frac{\pi}{2^{l-2}}, \frac{\pi}{2^{l-2}}+2 \alpha\right] ; \\
0, & \theta \in\left[\frac{\pi}{2^{l-2}}+2 \alpha, 2 \pi\right] .
\end{array}\right.
$$

Therefore, for any $l \in\{1, \cdots, L\}$

$$
\eta_{l, 1}(\theta)=\eta_{l, 0}\left(\theta-\frac{\pi}{2^{l-1}}\right)
$$

We shall now prove that, for any $l \in\{1, \cdots, L\}$

$$
\eta_{l, m}(\theta)=\eta_{l, 0}\left(\theta-\theta_{l, m}\right) \quad m=0,1, \cdots, 2^{l}-1 .
$$

Let's prove this by induction:

Now assume that for a fixed $l$ :

$$
\eta_{l, m}(\theta)=\eta_{l, 0}\left(\theta-\theta_{l, m}\right) \quad m=0,1, \cdots, 2^{l}-1
$$

with $\theta_{l, m}=m \frac{2 \pi}{2^{l}}$.

The inclusion of the induction hypothesis and Equation (22) in the expressions (12) and (13) gives:

$$
\begin{aligned}
\eta_{l+1,2 m}(\theta) & =\eta_{l, m}(\theta) \tau_{\alpha}\left(\theta-\pi-\frac{(2 m+1) \pi}{2^{l}}\right) \\
& =\eta_{l, 0}\left(\theta-\theta_{l, m}\right) \tau_{\alpha}\left(\theta-\pi-\theta_{l, m}-\frac{\pi}{2^{l}}\right) \\
& =\eta_{l, 0}\left(\theta-\theta_{l+1,2 m}\right) \tau_{\alpha}\left(\theta-\pi-\theta_{l+1,2 m}-\frac{\pi}{2^{l}}\right) \\
& =\eta_{l+1,0}\left(\theta-\theta_{l+1,2 m}\right), \\
\eta_{l+1,2 m+1}(\theta) & =\eta_{l, m}(\theta) \tau_{\alpha}\left(\theta-\frac{(2 m+1) \pi}{2^{l}}\right) \\
& =\eta_{l, 0}\left(\theta-\theta_{l, m}\right) \tau_{\alpha}\left(\theta-\theta_{l, m}-\frac{\pi}{2^{l}}\right) \\
& =\eta_{l, 0}\left(\theta-\theta_{l+1,2 m}\right) \tau_{\alpha}\left(\theta-\theta_{l+1,2 m}-\frac{\pi}{2^{l}}\right) \\
& =\eta_{l+1,1}\left(\theta-\theta_{l+1,2 m}\right)=\eta_{l+1,0}\left(\theta-\theta_{l+1,2 m+1}\right) .
\end{aligned}
$$

The proposition shows that for each level of construction $l$, the functions $\eta_{l, m}$ are continuous with compact support of size $\frac{2 \pi}{2^{l}}+2 \alpha$. So the aperture of the cone in frequency space supporting of $\hat{\psi}_{j, n, l, m}$ is equal to $\frac{2 \pi}{2^{l}}+2 \alpha$. Therefore, the contourlets $\psi_{j, n, l, m}$ are directional [26,27], and the angular selectivity of these new contourlets is proportional to $2^{l}$. Keeping that in mind, we will call the new contourlets $\psi_{j, n, l, m}$ the multiselective contourlets, and the parameter $l$ the angular selectivity level.

The central result is that for each selectivity level $l$, the multiselective contourlets generate a tight frame for each subspace $W_{j}$.

Theorem 3.1 for any $l \in\{0, \cdots, L\}$ the family $\left\{\psi_{j, n, l, m}: n \in \square^{2}, m=0,1, \cdots, 2^{l}-1\right\}$ is a tight frame for $W_{j}$.

\section{Proof}

To prove that the family $\left\{\psi_{j, n, l, m}: n \in \square^{2}, m=0,1, \cdots, 2^{l}-1\right\}$ is a tight frame for $W_{j}$, it suffices to evaluate the equality:

$$
\sum_{n \in \square^{2}} \sum_{m=0}^{2^{l}-1}\left|\left\langle\psi_{j, n, l, m} \mid f\right\rangle\right|^{2}=\|f\|^{2}, \quad \forall f \in W_{j} .
$$

Define the quantities

$$
E(f)=\sum_{n \in \square^{2}} \sum_{m=0}^{2^{l}-1}\left|\left\langle\psi_{j, n, j, l, m} \mid f\right\rangle\right|^{2},
$$

and

$$
\Delta\left(\psi_{j, n, l, m}\right)(\boldsymbol{k})=\hat{\psi}_{j, l, m}(\boldsymbol{k}) \hat{\psi}_{j, l, m}^{\star}\left(\boldsymbol{k}-2^{j} n\right) .
$$

Let us prove first that 


$$
\begin{aligned}
& E(f) \\
& =4 \pi^{2} \sum_{n \in \square^{2}} \int_{\mathbb{R}^{2}} \sum_{m=0}^{2^{l}-1} \Delta\left(\psi_{j, n, l, m}\right)(\boldsymbol{k}) \hat{f}(\boldsymbol{k}) \hat{f}^{\star}\left(\boldsymbol{k}-2^{j} n\right) \mathrm{d} \boldsymbol{k} .
\end{aligned}
$$

We have

$$
\left|\left\langle\psi_{j, n, j, l, m} \mid f\right\rangle\right|^{2}
$$$$
=\int_{\mathbb{R}^{2}} f(\boldsymbol{x}) \psi_{j, n, l, m}^{\star}(\boldsymbol{x}) \mathrm{d} \boldsymbol{x} \int_{\mathbb{R}^{2}} f^{\star}\left(\boldsymbol{x}^{\prime}\right) \psi_{j, n, l, m}\left(\boldsymbol{x}^{\prime}\right) \mathrm{d} \boldsymbol{x}^{\prime}
$$$$
=\int_{\mathbb{R}^{2}} f(x) \psi_{j, l, m}^{\star}\left(x-2^{j} n\right) \mathrm{d} \boldsymbol{x}
$$$$
\cdot \int_{\mathbb{R}^{2}} f^{\star}\left(\boldsymbol{x}^{\prime}\right) \psi_{j, l, m}\left(\boldsymbol{x}^{\prime}-2^{j} n\right) \mathrm{d} \boldsymbol{x}^{\prime}
$$$$
=\int_{\mathbb{R}^{2}} \mathrm{e}^{\mathrm{i}^{j}{ }^{j} \boldsymbol{k}} \hat{\psi}_{j, l, m}^{\star}(\boldsymbol{k}) \hat{f}(\boldsymbol{k}) \mathrm{d} \boldsymbol{k}
$$$$
\cdot \int_{\mathbb{R}^{2}} \mathrm{e}^{\mathrm{i} 2^{j} n \boldsymbol{k}^{\prime}} \hat{\psi}_{j, l, m}\left(\boldsymbol{k}^{\prime}\right) \hat{f}^{\star}\left(\boldsymbol{k}^{\prime}\right) \mathrm{d} \boldsymbol{k}^{\prime}
$$$$
=\int_{\mathbb{R}^{2}} \int_{\mathbb{R}^{2}} \mathrm{e}^{\mathrm{i} 2^{j} n\left(\boldsymbol{k}-\boldsymbol{k}^{\prime}\right)} \hat{\psi}_{j, l, m}(\boldsymbol{k}) \hat{\psi}_{j, l, m}^{\star}\left(\boldsymbol{k}^{\prime}\right) \hat{f}(\boldsymbol{k}) \hat{f}^{\star}\left(\boldsymbol{k}^{\prime}\right) \mathrm{d} \boldsymbol{k} \mathrm{d} \boldsymbol{k}^{\prime} .
$$

Using the Poisson formula

$$
\sum_{n \in \square^{2}} \mathrm{e}^{\mathrm{i} 2^{j} n\left(\boldsymbol{k}-\boldsymbol{k}^{\prime}\right)}=4 \pi^{2} \sum_{n \in \square^{2}} \delta\left(\boldsymbol{k}-\boldsymbol{k}-2^{j} n\right) .
$$

We obtain

$$
\begin{aligned}
& E(f) \\
& =4 \pi^{2} \sum_{n \in \square^{2}} \int_{\mathbb{R}^{2}} \sum_{m=0}^{2^{l}-1} \Delta\left(\psi_{j, n, l, m}\right)(\boldsymbol{k}) \hat{f}(\boldsymbol{k}) \hat{f}^{\star}\left(\boldsymbol{k}-2^{j} n\right) \mathrm{d} \boldsymbol{k} .
\end{aligned}
$$

We shall now prove that

$$
\sum_{m=0}^{2^{l}-1} \Delta\left(\psi_{j, n, l, m}\right)(\boldsymbol{k})=\hat{\psi}_{j}(\boldsymbol{k}) \hat{\psi}_{j}^{\star}\left(\boldsymbol{k}-2^{j} n\right) .
$$

According to the property (10), we verify that

$$
\tau_{\alpha}^{2}(\theta)+\tau_{\alpha}^{2}(\theta-\pi)=1
$$

Hence, for any $l \in\{0, \cdots, L\}$

$$
\begin{aligned}
& \sum_{m=0}^{2^{l}-1} \Delta\left(\psi_{j, n, l, m}\right)(\boldsymbol{k}) \\
& =\sum_{m=0}^{2^{l-1}-1}\left[\left(\psi_{j, l, 2 m}\right)(\boldsymbol{k}) \hat{\psi}_{j, l, 2 m}^{\star}\left(\boldsymbol{k}-2^{j} n\right)\right. \\
& \left.+\left(\psi_{j, l, 2 m+1}\right)(\boldsymbol{k}) \hat{\psi}_{j, l, 2 m+1}^{\star}\left(\boldsymbol{k}-2^{j} n\right)\right] \\
& =\sum_{m=0}^{2^{l-1}-1}\left[\hat{\psi}_{j, l-1, m}(\boldsymbol{k}) \hat{\psi}_{j, l-1, m}^{\star}\left(\boldsymbol{k}-2^{j} n\right) \tau_{\alpha}^{2}\left(\bar{\theta}_{m, l}\right)\right. \\
& \left.+\hat{\psi}_{j, l-1, m}(\boldsymbol{k}) \hat{\psi}_{j, l-1, m}^{\star}\left(\boldsymbol{k}-2^{j} n\right) \tau_{\alpha}^{2}\left(\bar{\theta}_{m, l}-\pi\right)\right] \\
& =\sum_{m=0}^{2^{l-1}-1} \hat{\psi}_{j, l-1, m}(\boldsymbol{k}) \hat{\psi}_{j, 0, l-1, m}^{\star}\left(\boldsymbol{k}-2^{j} n\right) \\
& \cdot\left[\tau_{\alpha}^{2}\left(\bar{\theta}_{m, l}\right)+\tau_{\alpha}^{2}\left(\bar{\theta}_{m, l}-\pi\right)\right] \\
& =\sum_{m=0}^{2^{l-1}-1} \hat{\psi}_{j, l-1, m}(\boldsymbol{k}) \hat{\psi}_{j, l-1, m}^{\star}\left(\boldsymbol{k}-2^{j} n\right)=\sum_{m=0}^{2^{l-1}-1} \Delta\left(\psi_{j, n, l-1, m}\right)(\boldsymbol{k}),
\end{aligned}
$$

with $\bar{\theta}_{m, l}=\theta-\frac{(2 m+1) \pi}{2^{l-1}}$.

Therefore,

$$
\sum_{m=0}^{2^{l}-1} \Delta\left(\psi_{j, n, l, m}\right)(\boldsymbol{k})=\Delta\left(\psi_{j, n, 0,0}\right)(\boldsymbol{k})=\hat{\psi}_{j}(\boldsymbol{k}) \hat{\psi}_{j}^{\star}\left(\boldsymbol{k}-2^{j} n\right)
$$

The equalities (8), (26), (28) and (30) imply that

$$
\begin{aligned}
& \sum_{n \in \square^{2}} \sum_{m=0}^{2^{l}-1}\left|\left\langle\psi_{j, n, l, m} \mid f\right\rangle\right|^{2} \\
& =4 \pi^{2} \sum_{n \in \square^{2}} \int_{\mathbb{R}^{2}} \hat{\psi}_{j}(\boldsymbol{k}) \hat{\psi}_{j}^{\star}\left(\boldsymbol{k}-2^{j} n\right) \hat{f}_{j}(\boldsymbol{k}) \hat{f}_{j}^{\star}\left(\boldsymbol{k}-2^{j} n\right) \mathrm{d} \boldsymbol{k} \\
& =\sum_{n \in \square^{2}} \int_{\mathbb{R}^{2}} \mathrm{e}^{\mathrm{i} 2^{j} n \boldsymbol{\psi}} \hat{\psi}_{j}^{\star}(\boldsymbol{k}) \hat{f}(\boldsymbol{k}) \mathrm{d} \boldsymbol{k} \int_{\mathbb{R}^{2}} \mathrm{e}^{\mathrm{i} 2^{j} n \boldsymbol{k}^{\prime}} \hat{\psi}_{j}\left(\boldsymbol{k}^{\prime}\right) \hat{f}^{\star}\left(\boldsymbol{k}^{\prime}\right) \mathrm{d} \boldsymbol{k}^{\prime} \\
& =\sum_{n \in \square^{2}} \int_{\mathbb{R}^{2}} f(\boldsymbol{x}) \psi_{j, n}^{\star}(\boldsymbol{x}) \mathrm{d} \boldsymbol{x} \int_{\mathbb{R}^{2}} f^{\star}\left(\boldsymbol{x}^{\prime}\right) \psi_{j, n}\left(\boldsymbol{x}^{\prime}\right) \mathrm{d} \boldsymbol{x}^{\prime} \\
& =\sum_{n \in \square^{2}}\left|\left\langle\psi_{j, n,} \mid f\right\rangle\right|^{2}=\|f\|^{2} .
\end{aligned}
$$

Therefore, for each selectivity level $l$, any function $f \in W_{j}$ is represented as:

$$
f(x)=\sum_{n \in \square^{2}} \sum_{m=0}^{l^{2}-1}\left\langle\psi_{n, j, l, m} \mid f\right\rangle \psi_{j, n, l, m}(x) .
$$

Since $V_{j_{0}}=V_{J} \oplus\left(\oplus_{j=j_{0}}^{J} W_{j}\right)$, any $f \in V_{j_{0}}$ is represented as:

$$
\begin{aligned}
f(\boldsymbol{x}) & =\sum_{n \in \square^{2}} \zeta_{J, n} \phi_{J, n}(\boldsymbol{x}) \\
& +\sum_{n \in \square^{2}} \sum_{j=j_{0}}^{J} \sum_{m=0}^{l^{2}-1} \omega_{j, n, l, m} \psi_{j, n, l, m}(\boldsymbol{x}),
\end{aligned}
$$

with

$$
\begin{aligned}
& \zeta_{J, n}=\left\langle\phi_{J, n} \mid f\right\rangle, \\
& \omega_{j, n, l, m}=\left\langle\psi_{j, n, l, m} \mid f\right\rangle,
\end{aligned}
$$

and the decompositions of $L^{2}\left(\mathbb{R}^{2}\right)$ into mutual orthogonal subspaces:

$$
L^{2}\left(\square^{2}\right)=V_{J} \oplus\left(\bigoplus_{j \leq J} W_{j}\right),
$$

imply that for each selectivity level $l$, the the family $\left\{\phi_{J, n}, \psi_{j, n, l, m}: n \in \square^{2}, \quad j \leq J, m=0,1, \cdots, 2^{l}-1\right\}$ is a tight frame for $L^{2}\left(\mathbb{R}^{2}\right)$, on which any function $f \in L^{2}\left(\mathbb{R}^{2}\right)$ is represented as:

$$
\begin{aligned}
f(\boldsymbol{x}) & =\sum_{n \in \square^{2}} \zeta_{J, n} \phi_{J, n}(\boldsymbol{x}) \\
& +\sum_{n \in \square^{2}} \sum_{j \leq J} \sum_{m=0}^{l^{2}-1} \omega_{j, n, l, m} \psi_{j, n, l, m}(\boldsymbol{x})
\end{aligned}
$$


The multiselective decomposition of $f \in L^{2}\left(\mathbb{R}^{2}\right)$ is defined as the set of the coefficients $\omega_{j, n, l, m}$ up to a scale $J$ and a selectivity level $L$ plus the remaining low-frequency information $\zeta_{J, n}$ :

$$
\left[\left\{\omega_{j, n, l, m}\right\}_{j \leq J, n \in \square^{2}, 0 \leq m<2^{l}, 0 \leq l \leq L},\left\{\zeta_{J, n}\right\}_{n \in \square^{2}}\right] .
$$

Since the multiselective contourlets decompose the image with the different selectivity level $l \in\{0,1, \cdots, L\}$, this multiselective decomposition represents and captures different structures of the image geometry. In particular, for each level $l \in\{0,1, \cdots, L\}$, theorem 2.1 shows that the multiselective contourlets $\psi_{j, n, l, m}$ generate a tight frame, on which we can reconstruct the original image according to (37). But there is more. Indeed, as shown in the following proposition, we can mix different frames inside the same reconstruction formula.

Proposition 3.2 for any function:

$$
\ell: \begin{aligned}
& \mathbb{R}^{2} \times\left[j_{0}, J\right] \rightarrow \mathbb{N} \\
& (\boldsymbol{x}, j) \mapsto \ell(\boldsymbol{x}, j)
\end{aligned},
$$

we obtain the following reconstruction for any $f \in v_{j_{0}}$

$$
\begin{aligned}
f(\boldsymbol{x}) & =\sum_{n \in \square^{2}} \zeta_{J, n} \phi_{J, n}(\boldsymbol{x}) \\
& +\sum_{j=j_{0}}^{J} \sum_{n \in \square^{2}} \sum_{m=0}^{\ell^{2}-1} \omega_{j, n, \ell, m} \psi_{j, n, \ell, m}(\boldsymbol{x}),
\end{aligned}
$$

with

$$
\begin{aligned}
& \zeta_{J, n}=\left\langle\phi_{J, n} \mid f\right\rangle, \\
& \omega_{j, n, \ell, m}=\left\langle\psi_{j, n, \ell, m} \mid f\right\rangle .
\end{aligned}
$$

\section{Proof}

Define the quantity

$$
\Upsilon(f)(x)=\sum_{n \in \square^{2}} \sum_{m=0}^{2^{\ell}-1} \omega_{j, n, \ell, m} \psi_{j, n, \ell, m}(x) .
$$

We shall now prove that, for any $\ell \in \mathbb{N}$ :

$$
\Upsilon(f)(x)=\sum_{n \in \square^{2}} \omega_{j, n} \psi_{j, n}(x) .
$$

We have

$$
\begin{aligned}
& \omega_{j, n, \ell, m} \psi_{j, n, \ell, m}(x) \\
& =\left\langle\psi_{j, n, \ell, m} \mid f\right\rangle \psi_{j, n, \ell, m}(\boldsymbol{x}) \\
& =\int_{\mathbb{R}^{2}} f\left(\boldsymbol{x}^{\prime}\right) \psi_{n, j, \ell, m}^{\star}\left(\boldsymbol{x}^{\prime}\right) \mathrm{d} \boldsymbol{x}^{\prime} \psi_{n, j, \ell, m}(\boldsymbol{x}) \\
& =\int_{\mathbb{R}^{2}} f\left(\boldsymbol{x}^{\prime}\right) \psi_{j, \ell, m}^{\star}\left(\boldsymbol{x}^{\prime}-2^{j} n\right) \mathrm{d} \boldsymbol{x}^{\prime} \psi_{j, \ell, m}\left(\boldsymbol{x}^{\prime}-2^{j} n\right) \\
& =\int_{\mathbb{R}^{2}} \mathrm{e}^{\mathrm{i} 2^{j} n \boldsymbol{k}^{\prime}} \hat{\psi}_{j, \ell, m}^{\star}\left(\boldsymbol{k}^{\prime}\right) \hat{f}\left(\boldsymbol{k}^{\prime}\right) \mathrm{d} \boldsymbol{k}^{\prime} \int_{\mathbb{R}^{2}} \mathrm{e}^{\mathrm{i}\left(x-2^{j} n\right) \boldsymbol{k}} \hat{\psi}_{j, \ell, m}(\boldsymbol{k}) \mathrm{d} \boldsymbol{k} \\
& =\int_{\mathbb{R}^{2}} \mathrm{e}^{\mathrm{i} 2{ }^{j} n \boldsymbol{k}^{\prime}} \hat{\psi}_{j, \ell, m}^{\star}\left(\boldsymbol{k}^{\prime}\right) \hat{f}\left(\boldsymbol{k}^{\prime}\right) \mathrm{d} \boldsymbol{k}^{\prime} \int_{\mathbb{R}^{2}} \mathrm{e}^{\mathrm{i} 2^{j} n \boldsymbol{k}} \hat{\psi}_{j, \ell, m}(\boldsymbol{k}) \mathrm{e}^{-\mathrm{i} \boldsymbol{x} \boldsymbol{d}} \mathrm{d} \boldsymbol{k} \\
& =\int_{\mathbb{R}^{2}} \int_{\mathbb{R}^{2}} \mathrm{e}^{\mathrm{i} 2{ }^{j} n\left(\boldsymbol{k}^{\prime}-\boldsymbol{k}\right)} \hat{\psi}_{j, \ell, m}^{\star}\left(\boldsymbol{k}^{\prime}\right) \hat{\psi}_{j, \ell, m}(\boldsymbol{k}) \hat{f}\left(\boldsymbol{k}^{\prime}\right) \mathrm{e}^{-\mathrm{i} \boldsymbol{x} k} \mathrm{~d} \boldsymbol{k}^{\prime} \mathrm{d} \boldsymbol{k} .
\end{aligned}
$$

Using the Poisson formula

$$
\sum_{n \in \square^{2}} \mathrm{e}^{\mathrm{i} 2^{j} n\left(\boldsymbol{k}^{\prime}-\boldsymbol{k}\right)}=4 \pi^{2} \sum_{n \in \square^{2}} \delta\left(\boldsymbol{k}^{\prime}-\boldsymbol{k}-2^{j} n\right) .
$$

and the equality (30), we obtain:

$$
\begin{aligned}
& \Upsilon(f)(x) \\
& =4 \pi^{2} \sum_{n \in \square^{2}} \sum_{m=0}^{2^{\ell}-1} \int_{\mathbb{R}^{2}} \hat{\psi}_{j, \ell, m}^{\star}\left(\boldsymbol{k}^{\prime}\right) \hat{\psi}_{j, \ell, m}\left(\boldsymbol{k}^{\prime}-2^{j} n\right) \hat{f}\left(\boldsymbol{k}^{\prime}\right) \\
& \cdot \mathrm{e}^{-\mathrm{i} x\left(\boldsymbol{k}^{\prime}-2^{j} n\right)} \mathrm{d} \boldsymbol{k}^{\prime} \\
& =4 \pi^{2} \sum_{n \in \square^{2}} \int_{\mathbb{R}^{2}}\left[\sum_{m=0}^{2^{\ell}-1} \hat{\psi}_{j, \ell, m}^{\star}\left(\boldsymbol{k}^{\prime}\right) \hat{\psi}_{j, \ell, m}\left(\boldsymbol{k}^{\prime}-2^{j} n\right)\right] \hat{f}\left(\boldsymbol{k}^{\prime}\right) \\
& \cdot \mathrm{e}^{-\mathrm{i} x\left(\boldsymbol{k}^{\prime}-2^{j} n\right)} \mathrm{d} \boldsymbol{k}^{\prime} \\
& =4 \pi^{2} \sum_{n \in \square^{2}} \int_{\mathbb{R}^{2}}\left[\hat{\psi}_{j}^{\star}\left(\boldsymbol{k}^{\prime}\right) \hat{\psi}_{j}\left(\boldsymbol{k}^{\prime}-2^{j} n\right)\right] \hat{f}\left(\boldsymbol{k}^{\prime}\right) \mathrm{e}^{-\mathrm{ix}\left(\boldsymbol{k}^{\prime}-2^{j} n\right)} \mathrm{d} \boldsymbol{k}^{\prime} \\
& =\sum_{n \in \square^{2}} \int_{\mathbb{R}^{2}} \mathrm{e}^{\mathrm{i} 2^{j} n \boldsymbol{k}} \hat{\psi}_{j}^{\star}\left(\boldsymbol{k}^{\prime}\right) \hat{f}\left(\boldsymbol{k}^{\prime}\right) \mathrm{d} \boldsymbol{k}^{\prime} \int_{\mathbb{R}^{2}} \mathrm{e}^{-\mathrm{i} 2^{j} n \boldsymbol{k}} \hat{\psi}_{j}(\boldsymbol{k}) \mathrm{e}^{-\mathrm{i} \boldsymbol{x} \boldsymbol{k}} \mathrm{d} \boldsymbol{k} \\
& =\sum_{n \in \square^{2}} \int_{\mathbb{R}^{2}} \mathrm{e}^{\mathrm{i} 2^{j} n \boldsymbol{k}} \hat{\psi}_{j}^{\star}\left(\boldsymbol{k}^{\prime}\right) \hat{f}\left(\boldsymbol{k}^{\prime}\right) \mathrm{d} \boldsymbol{k}^{\prime} \int_{\mathbb{R}^{2}} \mathrm{e}^{\mathrm{i}\left(x-2^{j} n\right) \boldsymbol{k}} \hat{\psi}_{j}(\boldsymbol{k}) \mathrm{d} \boldsymbol{k} \\
& =\sum_{n \in \square^{2}} \int_{\mathbb{R}^{2}} f\left(\boldsymbol{x}^{\prime}\right) \psi_{j}^{\star}\left(\boldsymbol{x}-2^{j} n\right) \mathrm{d} \boldsymbol{x}^{\prime} \psi_{j}\left(\boldsymbol{x}-2^{j} n\right) \\
& =\sum_{n \in \square^{2}} \int_{\mathbb{R}^{2}} f\left(\boldsymbol{x}^{\prime}\right) \psi_{j, n}^{\star}\left(\boldsymbol{x}^{\prime}\right) \mathrm{d} \boldsymbol{x}^{\prime} \psi_{j, n}(\boldsymbol{x}) \\
& =\sum_{n \in \square^{2}}\left\langle\psi_{j, n} \mid f\right\rangle \psi_{n, j}(\boldsymbol{x})=\sum_{n \in \square^{2}} \omega_{j, n} \psi_{j, n}(\boldsymbol{x}) .
\end{aligned}
$$

Since, we have for any $f \in v_{j_{0}}$

$$
f(\boldsymbol{x})=\sum_{n \in \square^{2}} \zeta_{J, n} \phi_{J, n}(\boldsymbol{x})+\sum_{j=j_{0}}^{J} \sum_{n \in \square^{2}} \omega_{j, n} \psi_{j, n}(\boldsymbol{x}) .
$$

we obtain the following reconstruction for any $f \in v_{j_{0}}$

$$
f(x)=\sum_{n \in \square^{2}} \zeta_{J, n} \phi_{J, n}(x)+\sum_{j=j_{0} n \in \square^{2}}^{J} \sum_{m=0}^{\ell^{2}-1} \omega_{j, n, \ell, m} \psi_{j, n, \ell, m}(x) .
$$

The reconstruction carried out in this proposition provides a new degree of freedom to describe images adaptively. Indeed, at each point $\boldsymbol{x} \in \mathbb{R}^{2}$ and each scale $j$, we may search the adaptive selectivity reconstruction, that is, the selectivity level $\ell(x, j)$ that improves the detection of the content of $f$.

\section{Image Super-Resolution via Multiselective Contourlets}

The main idea is similar to the technique of interpolation proposed in [18]. Our algorithm of image super-resolution is to alternately enforce two constraints.

\subsection{Anti-Aliasing Filer Constraint}

In wavelet-space extrapolation, the objective is to obtain 
an estimation $\hat{x}_{0}$ of high-resolution image $x$ from lowresolution image $x_{L}$ (refer to Figure 1). In this case we impose anti-aliasing filer constraint, that is the given low-resolution image is the downsampled output of the low-pass anti-aliasing filter in a wavelet transform. As a simple way to get an estimate $\hat{x}_{0}$ of the high resolution image, we can take the inverse wavelet transform by keeping $x_{L}$ as the low-pass band and zeropadding all high-pass subbands. Consequently, for any given image $y$, we can calculate the best approximation (in $L^{2}$ norm) to $y$, subject to anti-aliasing filer constraint, through orthogonal projection. Let $F$ and $F^{-1}$ represent the forward and inverse wavelet transforms, respectively; denote $P$ as the diagonal projection matrix of $1 \mathrm{~s}$ and $0 \mathrm{~s}$ that keeps the low-pass wavelet coefficients and zeros out the high frequency subband coefficients, and let $P^{\perp}=I-P$. If we use orthonormal wavelet transforms, then the projection of any image $y$ can be calculated by

$$
\hat{y}=F^{-1}\left(P^{\perp} F y+P F \hat{x}_{0}\right),
$$

where $\hat{x}_{0}$ is the estimation of the high-resolution image obtained as in Figure 1.

\subsection{Sparsity Constraint}

The second constraint is based on a model for natural images. Since the multiselective contourlets described in Section 3, generate a multiselective geometric representation well-suited to preserve contours and edges and geometric structure of image, we assume that the unknown high-resolution image should be sparse in the multiselective contourlets domain. For the sake of simplicity, we choose to use a direct hard-thresholding scheme in our proposed algorithm. Intuitively, we view our estimate to the high-resolution image as a noisy version of the true image. Enforcing our sparsity constraint works to denoise the estimation of the interpolated signal while retaining the important coefficients near edges. we enforce this constraint through a hard-thresholding of the multiselective contourlet coefficients.

We suppose that the estimation $\hat{x}$ of the highresolution is a multiresolution approximation of the real image $f$ at the resolution $2^{0}$. Hence $\hat{x} \in V_{0}$, and the multiselective contourlets decomposition $\mathcal{D}$ of $\hat{x}$ is defined as the set of the coefficients

$\omega_{j, n, l, m}=\left\langle\psi_{j, n, l, m} \mid \hat{x}\right\rangle$ up to a scale $J>0$ and a sele-

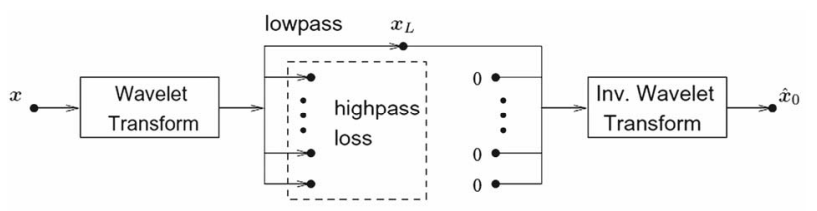

Figure 1. The anti-aliasing filer constraint. ctivity level $L>0$, plus the remaining low-frequency information $\zeta_{J, n}=\left\langle\phi_{J, n} \mid \hat{x}\right\rangle$ :

$$
\mathcal{D} \hat{x}=\left[\left\{\omega_{j, n, l, m}\right\}_{0<j \leq J, n \in \square^{2}, 0 \leq m<2^{l}, 0 \leq 1 \leq L},\left\{\zeta_{J, n}\right\}_{n \in \square^{2}}\right] .
$$

Denote $\mathcal{S}_{T}$ as the diagonal matrix that, given some threshold value $T$, zeros out insignificant coefficients in the coefficient vector whose absolute values are smaller than $T$; and $\mathcal{R}$ as the adaptive selectivity reconstruction given by proposition (3.2),

$$
\begin{aligned}
\mathcal{R} \hat{x}(\boldsymbol{t}) & =\sum_{n \in \square^{2}} \zeta_{J, n} \phi_{J, n}(\boldsymbol{t}) \\
& +\sum_{n \in \square^{2}} \sum_{j=1}^{J} \sum_{m=0}^{\ell^{2}-1} \omega_{j, n, \ell, m} \psi_{j, n, \ell, m}(\boldsymbol{t}) .
\end{aligned}
$$

we choice the adaptive selectivity level $\ell(j, t)$ by minimizing the distortion introduced by thresholding in fixed selectivity procedure:

$$
\ell(j, \boldsymbol{t})=\arg \min _{l \in[0, L]}\left|\sum_{n \in \square^{2}} \omega_{j, n, 0,0}(\boldsymbol{t})-\sum_{n \in \square^{2}} \bar{\omega}_{j, n, l, 0}(\boldsymbol{t})\right|,
$$

with

$$
\bar{\omega}_{j, n, l, 0}(\boldsymbol{t})=\sum_{m=0}^{l^{2}-1} \mathcal{S}_{T}\left(\omega_{j, n, l, m}\right) \psi_{j, n, l, m}(\boldsymbol{t}) .
$$

Denote $\tilde{X}$ the denoised high-resolution image. The sparseness constraint by hard-thresholding can be written as

$$
\tilde{x}=\mathcal{R} \mathcal{S}_{T} \mathcal{D} \hat{x}
$$

\subsection{Multiselective Contourlets Algorithm for Image Super-Resolution}

We show in Figure 2 the block diagram of the proposed multiselective contourlets algorithm for high-resolution image reconstruction, which can be summarized as follows:

1) We start our algorithm by taking $\hat{x}_{0}$, obtained by the simple wavelet interpolation shown in Figure 1, as the initial estimate of the high-resolution image.

2) We then attempt to improve the quality of interpolation, particularly in regions containing edges and contours, by iteratively enforcing the observation constraint as well as the sparseness constraint. Let $\hat{x}_{k}$ represent the estimate at the kth step. By combining (47) and (52), the new estimate $\hat{x}_{k+1}$ can then be obtained by

$$
\hat{x}_{k+1}=F^{-1}\left(P^{\perp} F \mathcal{R} \mathcal{S}_{T_{k}} \mathcal{D} \hat{x}_{k}+P F \hat{x}_{0}\right) .
$$

3) Following the same principle of the sparsenessbased image recovery algorithm proposed in [28], we gradually decrease the threshold value $T_{k}$ by a small amount $\delta$ in each iteration, i.e., $T_{k+1}=T_{k}-\delta$. This has been shown to be effective in circumventing the non- 


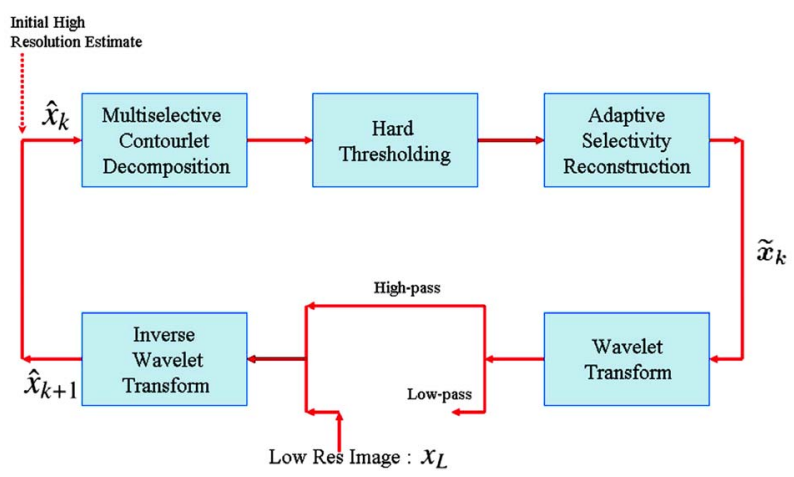

Figure 2. The block diagram of the proposed algorithm for image super-resolution.

convexity of the sparseness constraint.

4) Return to step 2 and keep iterating, until the generated images converge or a predetermined maximum iteration number has been reached.

\section{Numerical Experiments}

We compare the high-resolution images obtained by the proposed method with those obtained by wavelet linear [28], interpolation bicubic [29], contourlet transform [18], soft-decision adaptive interpolation (SAI) [16], and sparse mixing estimators (SME) [17]. In the experiments, we use five scales $J=5$, and five selectivity level $L=5$ for multiselective contourlets decomposition, and we choose $T_{0}=10$ and is decreased by $\delta=0.2$ in each iteration, with a maximum of 10 iterations. We use several standard test images of size $512 \times 512$, including Lenna, Boat, Gauss disc, Peppers, Straws, and Mandril (Figure 3). Gauss disc image includes regular regions, Lenna and Boat include both fine details and regular regions. Peppers is mainly composed of regular regions separated from sharp contours. Mandril is rich in fine details. Straws image contains directional patterns that are superposed in various directions. To show the true power of the interpolation algorithms, we first downsampled each image by a factor of 2 and then interpolated the result back to its original size.

The performance measure used was the Peak Signal to Noise Ratio (PSNR), A good high-resolution method must maximize the PSNR. Table 1 gives the PSNRs generated by all methods for the images in Figure 3. Figures 4 and 5 compare the high-resolution image obtained by different methods. Bicubic interpolations produce some blur and jaggy artifacts in the zoomed images, but the image quality is lower than with SME and SAI methods, as shown by the PSNRs. The Contourlet method yields almost the same PSNR as a bicubic interpolation but often provides better image quality. It is able to restore the geometrical structures (see Lenna's hat and gauss disc zoom) when the underlying contourlet

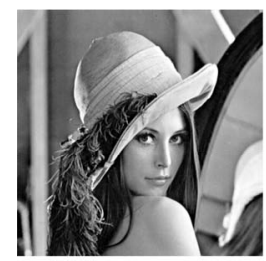

Lenna

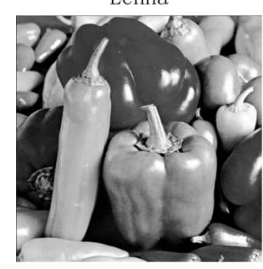

Peppers

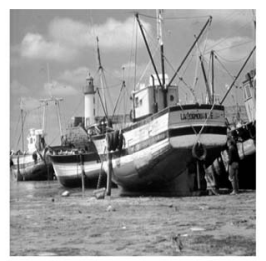

Boat

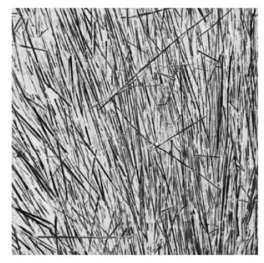

Straws

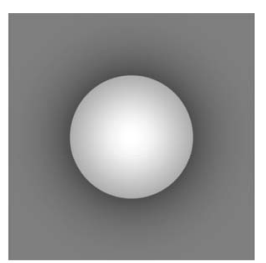

Gauss disc

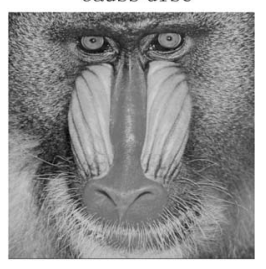

Mandri1
Figure 3. Images used in the numerical experiments.

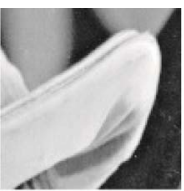

High-resolution

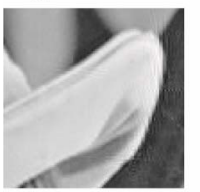

Contourlet

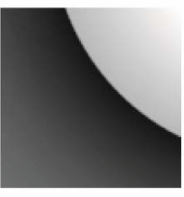

High-resolution

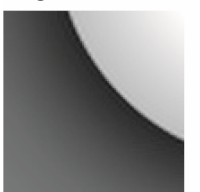

Contourlet

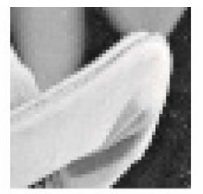

Low-resolution

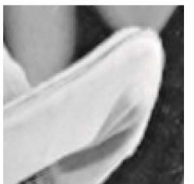

SME

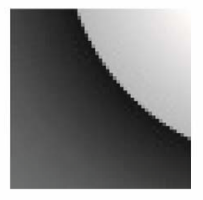

Low-resolution

SME

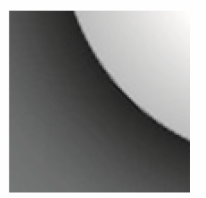

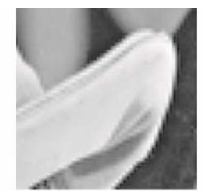

Wavelet linear

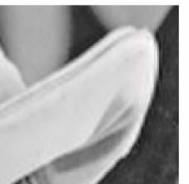

SAI

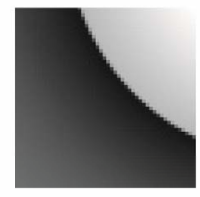

Waveletlinear

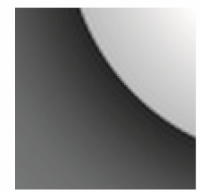

SAI

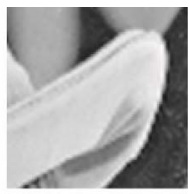

Bicubic

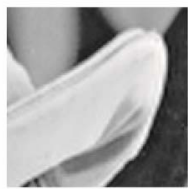

Proposed

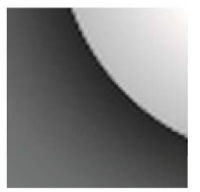

Bicubic

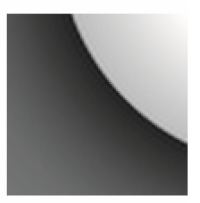

Proposed
Figure 4. The zoom-in comparison of the Lenna and Gauss disc images. From left to right: high-resolution image, lowresolution image (shown at the same scale by enlarging the pixel size), wavelet linear, bicubic interpolation, contourlet, SME, SAI, and proposed method.

vectors are accurately estimated. However, when the approximating contourlet vectors are not estimated correctly, it produces directional artifact patterns, because the contoulets represent the image geometry with the same angular selectivity. Contrariwise in our proposed method, the angular selectivity can be adapted locally to the content of the image, which improves its gain in PSNR and its regularity of object boundaries of geometrical structures in the generated images, as shown in Boat and Peppers zooms. 
Table 1. The performance of the proposed method relative to other methods. PSNRS (in decibels) are computed over images of Figure 3. From left to right: wavelet linear [28], interpolation bicubic [29], contourlet transform [18], sparse mixing estimators (SME) [17], and soft-decision adaptive interpolation (SAI) [16].

\begin{tabular}{|c|c|c|c|c|c|c|}
\hline Image & Wavelet lin & Bicubic & Contourlet & SME & SAI & Proposed \\
\hline Lenna & 31.59 & 34.03 & 34.17 & 34.61 & 34.74 & 35.10 \\
\hline Boat & 28.60 & 29.09 & 29.15 & 29.72 & 29.61 & 30.14 \\
\hline Gaussdisc & 42.86 & 46.88 & 48.45 & 50.61 & 50.46 & 50.89 \\
\hline Straws & 19.15 & 20.53 & 20.54 & 21.55 & 21.42 & 21.56 \\
\hline Mandril & 22.55 & 22.15 & 22.60 & 23.10 & 23.15 & 23.53 \\
\hline
\end{tabular}
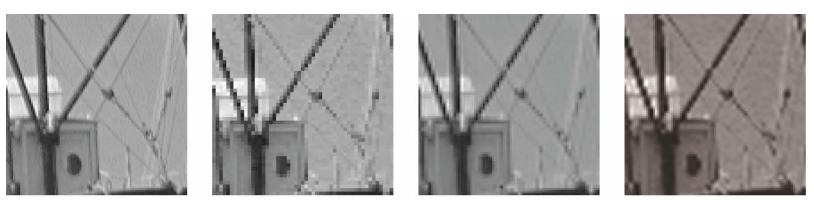

High-resolution

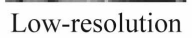

Waveletlinear

Bicubic
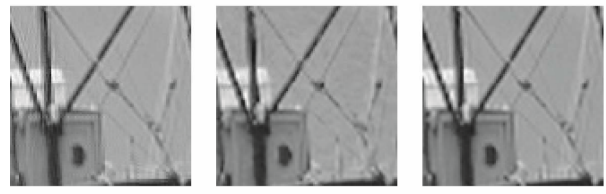

SME
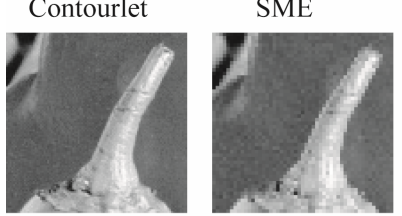

SAI
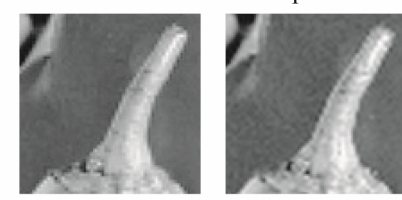

High-resolution

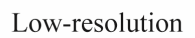

Waveletlinear

Bicubic

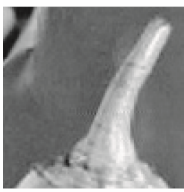

Contourlet

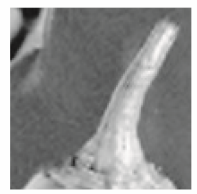

SME

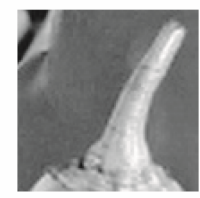

SAI

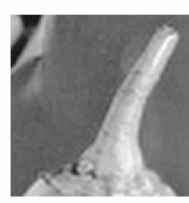

Proposed
Figure 5. The zoom-in comparison of the boat and peppers images. From left to right: high-resolution image, lowresolution image (shown at the same scale by enlarging the pixel size), wavelet linear, bicubic interpolation, contourlet, SME, SAI, and proposed method.

\section{Conclusion}

We have described a new method for high-resolution restoration of image using an iterative projection process based on anti-aliasing wavelet technique, and hard-thresholding scheme in a new multiselective contourlets analysis. This new multiselectve contourlets analysis can capture and restore slightly better regular geometrical structures of image. Experimental results show that the proposed algorithm achieves better super-resolution results than other super-resolution methods in the literature.

\section{REFERENCES}

[1] P. Milanfar, "Super-Resolution Imaging," CRC Press, 2011.

[2] D. Capel, "Image Mosaicing and Super-Resolution," Springer, Berlin, 2004. doi:10.1007/978-0-85729-384-8

[3] K. Katsaggelos, R. Molina and J. Mateos, "Super Resolution of Images and Video," Morgan and Claypool Publishers, San Rafael, 2007.

[4] S. Borman and R. L. Stevenson, "Super-Resolution from Image Sequences," Proceedings of the 1998 Midwest Symposium on Circuits and Systems, Vol. 2, 1998, pp. 374-378.

[5] S. Baker and T. Kanade, "Limits on Super-Resolution and How to Break Them," IEEE Transactions on Pattern Analysis and Machine Intelligence, Vol. 24, 2002, pp. 1167-1183. doi:10.1109/TPAMI.2002.1033210

[6] N. K. Bose S. P. Kim and H. M. Valenzuela, "Ecursive Reconstruction of High Resolution Image from Noisy Undersampled Multiframes," IEEE Transactions on Acoustics, Speech and Signal Processing, Vol. 38, 1990, pp. 1013-1027.

[7] R. Y. Tsai and T. S. Huang, "Multipleframe Image Restoration and Registration," Advances in Computer Vision and Image Processing, Greenwich, JAI Press Inc., 1984, pp. 317-339.

[8] E. Kaltenbacher and R. C. Hardie, "High-Resolution Infrared Image Reconstruction Using Multiple Low Resolution Aliased Frames," Proceedings of the IEEE National Aerospace Electronics Conference, Vol. 2, 1996, pp. 702709.

[9] A. K. Katsaggelos, B. C. Tom and N. P. Galatsanos, "Reconstruction of a High Resolution Image from Registration and Restoration of Low Resolution Images," Proceedings of the IEEE International Conference on Image Processing, Vol. 3, 1994, pp. 553-557.

[10] S. Chaudhuri and M. V. Joshi, "Motion-Free SuperResolution, MIT Press, Cambridge, 2005.

[11] R. Keys, "Cubic Convolution Interpolation for Digital Image Processing," IEEE Transactions on Acoustics, Speech and Signal Processing, Vol. 29, No. 6, 1981, pp. 1153-1160. doi:10.1109/TASSP.1981.1163711 
[12] M. Unser, "Splines: A Perfect Fit for Signal and Image Processing," IEEE Signal Processing Magazine, Vol. 16, No. 6, 1999, pp. 22-38. doi:10.1109/79.799930

[13] S. Carrato, G. Ramponi and S. Marsi, "A Simple Edge-Sensitive Image Interpolation Filter," Proceedings of IEEE International Conference on Image Processing, Lausanne, 16-19 September 1996, pp. 711-714.

[14] T. Lehmann, C. Gonner and K. Spitzer, "Survey: Interpolations Methods in Medical Image Processing," IEEE Transactions on Medical Imaging, Vol. 18, No. 11, 1999, pp. 1049-1075. doi:10.1109/42.816070

[15] P. Thevenaz, T. Blu and M. Unser, "Medical Images Application," IEEE Transactions on Medical Imaging, Vol. 19, 2000, pp. 739-758.

[16] X. Zhang and X. Wu, "Image Interpolation by Adaptive 2-D Autoregressive Modeling and Soft-Decision Estimation," IEEE Transactions on Image Processing, Vol. 17, No. 6, 2008, pp. 887-896. doi:10.1109/TIP.2008.924279

[17] S. Mallat and G. Yu, "Super-Resolution with Sparse Mixing Estimators," IEEE Transactions on Image Processing, Vol. 19, No. 11, 2010, pp. 518-531. doi:10.1109/TIP.2010.2049927

[18] N. Mueller, Y. Lu and M. N. Do, "Image Interpolation Using Multiscale Geometric Representations," Processing of SPIE Computational Imaging, Vol. 6498, San Jose, 28 January 2007.

[19] M. N. Do and M. Vetterli, "The Contourlet Transform: An Efficient Directional Multiresolution Image Representation," IEEE Transactions Image on Processing, Vol. 14, No. 12, 2005, pp. 2091-2106. doi:10.1109/TIP.2005.859376

[20] A. L. Cunha, J. Zhou and M. N. Do, "The Nonsubsampled Contourlet Transform: Theory, Design, and Applications," IEEE Transactions Image on Processing, Vol. 15, No. 10, 2006, pp. 3089-3101. doi:10.1109/TIP.2006.877507
[21] J. P. Antoine, R. Murenzi, P. Vandergheynst and S. T. Ali, "Two Dimensional Wavelets and Their Relatives," Cambridge University Press, Cambridge, 2004. doi:10.1017/CBO9780511543395

[22] P. J. Burt and E. H. Adelson, "The Laplacian Pyramid as a Compact Image Code," IEEE Transactions on Communications, Vol. 31, No. 4, 1983, pp. 532-540. doi:10.1109/TCOM.1983.1095851

[23] M. Vetterli and J. Kovacevic, "Wavelets and Subband Coding," Prentice Hall, Upper Saddle River, 1995.

[24] S. Mallat, "A Wavelet Tour of Signal Processing," 2nd Edition, Academic Press, Waltham, 1999.

[25] M. N. Do and M. Vetterli, "Framing Pyramids," IEEE Transactions on Signal Processing, Vol. 51, No. 9, 2003, pp. 2329-2342.

[26] J. P. Antoine, R. Murenzi and P. Vandergheynst, "Directional Wavelets Revisited: Cauchy Wavelets and Symmetry Etection in Patterns," Applied and Computational Harmonic Analysis, Vol. 6, No. 3, 1999, pp. 314-345. doi:10.1006/acha.1998.0255

[27] M. El Aallaoui, A. El Bouhtouri and A. Ayadi, "Adaptive Selectivity Representation: Design, and Applications," International Journal of Wavelets, Multiresolution and Information Processing, Vol. 7, No. 1, 2009, pp. 89-113. doi:10.1142/S0219691309002829

[28] O. G. Guleryuz, "Nonlinear Approximation Based Image Recovery Using Adaptive Sparse Reconstructions and Iterated Denoising: Part I-Theory," IEEE Transactions on Image Processing, Vol. 15, No. 3, 2006, pp. 539-554. doi:10.1109/TIP.2005.863057

[29] T. Blu A. Muoz and M. Unser. Least-Squares Image Resizing Using Finite Differences," IEEE Transactions on Image Processing, Vol. 10, No. 9, 2001, pp. 1365-1378. doi:10.1109/83.941860 